\title{
A Weighted ML-KNN Model for Predicting Users' Personality Traits
}

\author{
Xianglin Zuo ${ }^{1, \mathrm{a}}$,Baoping Feng ${ }^{1, \mathrm{~b}}$,Yuan $\mathrm{Yao}^{1, \mathrm{c}}$,Tianyi Zhang ${ }^{1, \mathrm{~d}}$, Qian Zhang ${ }^{1, \mathrm{e}}$, \\ Mengmeng Wang ${ }^{1, f}$ and Wanli Zuo ${ }^{1, g}$ \\ ${ }^{1}$ College of Computer Science and Technology, Jilin University, Changchun 130012, P.R.China \\ a295228473@qq.com, b1040791153@qq.com, '912244016@qq.com, d1040791153@qq.com, \\ e912244016@qq.com, 'wmmwwlh@126.com, ${ }^{\mathrm{g}}$ wanli@jlu.edu.cn
}

Keywords: Big-5 personality traits; personality prediction; ML-kNN; weighted feature; information entropy theory

\begin{abstract}
Gaining insight into human personality and its impact on human behavior is very valuable in many applications, such as web information credibility prediction. In this paper, we explore using weighted ML-kNN model for automatic recognition of personality traits of web users, based on a given composition text. After extracting features through analysis of the content of user's Essays statues updates, we discretize contiguous attribute using Kohonen's feature-map algorithm, and assign weight to extracted features based on information entropy. The Essays dataset is partitioned into training dataset and test dataset. For a given test user, the weighted distance between test user and training user is calculated, and based on which the nearest neighbors are identified. The personality traits of test user are then predicted by using ML-kNN algorithm. Our experiment on the Essays dataset shows expected positive results.
\end{abstract}

\section{Introduction}

Social networks have become a most popular mediums for information dissemination as well as facilitators of social interactions [1]. Gaining insight into human personality and its impact on human behavior is very valuable in many respects [2]. Social connections among individuals can yield richer information than his/her isolated attributes [3]. Consequently, it is necessary to understand human behavior for the natural of social interactions.

In the Workshop on Computational Personality Recognition (Shared Task), different systems for personality recognition from text on a common benchmark have been compared. Considering the results on Facebook train-test split as a proof of concept, B. Verhoeven et al proposed a meta-learning approach which can be extended to certain component classifiers from other genres with other class systems, or even from other languages [4]. G. Farnadi et.al explored the use of ML techniques (SVM,kNN,NB) for automatic recognition of personality traits from users' Facebook statues updates [5], which outperform the majority class baseline algorithms even with a small set of training dataset. [6] achieved a high performance using ranking algorithms for feature selection, SVMs and Boosting (B) as learning algorithms. F. Alam et al explored the suitability and performance of several classification techniques based on a set of features extracted from Facebook data [2]. For automatic recognition, [1] studied different classification methods such as SMO, BLR and MNB. [7] employed features in five SVM classifiers for detecting five personality traits through Essays. D.S. Appling et al modeled dependencies between different personality traits using conditional random fields. [8,9] performed regression analysis to identify significant correlations between personality dimensions on the Big-5 Personality inventory and speech act labeling.

In this paper, we present a weighted ML-kNN model for predicting an individual's BIG-5 personality traits based on analysis of the content of his/her Essays text. At firstly, we extract features by analyzing the content of user's Essays statues updates. Then, we discretize the linguistic and emotional features of content that users produced on the basis of Kohonen's feature-maps algorithm. After that, we assign weights to extracted features based on information entropy theory. Finally, we apply weighted ML-kNN model to predict users' personality traits based on weighted feature set. We 
run a set of experiments to investigate the performance of our model at predicting web user personality traits, and report system performances in terms of precision, recall and F1-meature.

\section{Method}

Extracting Features. The features for predicting uses' personality are complex, in this paper we mainly consider two aspects: linguistic features and emotional features which are based on linguistic features.

(1) Linguistic Features. In order to learn traits of content that user yields, we get word frequency statistics of 34 kinds of parts of speech with Stanford Parser (http://nlp.stanford.edu/software/ lex-parser.shtml\#About): CC (conjunction, coordination), CD (numeral, cardinal), DT (determiner), $E X$ (existential there), $F W$ (foreign word), IN (preposition or conjunction, subordinating), $J J$ (adjective or numeral, ordinal), JJR (adjective, comparative), JJS (adjective, superlative), $N N$ (noun, common, singular or mass), NNS (noun, common, plural), NNP (noun, proper, singular), NNPS (noun, proper, plural), $P D T$ (pre-determiner), $P O S$ (genitive marker), $P R P$ (pronoun, personal), $R B$ (adverb), $R B R$ (adverb, comparative), RBS (adverb, superlative), RP (particle), SYM (symbol), UH (interjection), $V B$ (verb, base form), $V B D$ (verb, past tense), $V B G$ (verb, present participle or gerund), $V B N$ (verb, past participle), VBP (verb, present tense, not 3rd person singular), WDT (WH-determiner), WP (WH-pronoun), WRB (WH-adverb), comma, period, exclamation and question.

(2) Emotional Features. Based on the corpus for sentiment analysis of HowNet Knowledge (http://www.keenage.com/download/sentiment.rar),we extract emotional words whose part-of-speech tag is JJ (adjective or numeral, ordinal), JJR (adjective, comparative), JJS (adjective, superlative), RB (adverb), RBR (adverb, comparative) or RBS (adverb, superlative).

The calculation formulas of user,'s positive, negative and neutral emotional features scores are shown in Eq.1, Eq.2, Eq3 respectively.

$$
\begin{aligned}
& \text { Positive }_{i}=\mid \text { Positive words }\left.\right|_{i} / \mid \text { Emotional words }\left.\right|_{i} \text {, } \\
& \text { Negative }_{i}=\mid \text { Negative } \text { words }\left.\right|_{i} / \mid \text { Emotional words }\left.\right|_{i} \text {, } \\
& \text { Neutral }_{i}=1-\text { Positive }_{i}-\text { Negative }_{i},
\end{aligned}
$$

where $\mid$ Emotional words $\left.\right|_{i}$ represents the number of emotional words which user ${ }_{i}$ uses in his/her contents; $\mid$ Positive words $\left.\right|_{i}$ and $\mid$ Negative words $\left.\right|_{i}$ represent the number of positive words and the number of negative words among all emotional words which user ${ }_{i}$ uses respectively.

Discretizing Attribute Values. We partition contiguous attribute values into intervals based on Kohonen's feature-maps algorithm, the purpose of which is to assign weight to each attribute using fuzzy information entropy theory.

Calculating weighted $k$ Nearest Neighbors. ML-kNN Model is derived from the traditional $k$-Nearest Neighbor $(\mathrm{kNN})$ algorithm. For each unseen instance, its $k$ nearest neighbors in the training set are firstly identified. For this purpose, we calculate the distance based on weighted features.

According to the different correlations between features and users' personality, we compute weight of each feature based on information entropy theory, in order to calculate the distance between two users. The calculation formula of feature $f_{j}$ 's weight is shown in Eq. 4.

$$
\text { Weight }_{j}=I G\left(f_{j}\right) / \sum_{h=1}^{r} I G\left(f_{h}\right) \text {, }
$$

where $\operatorname{IG}\left(f_{j}\right)$ represents information gain of feature $f_{j}$ which is shown in Eq. 5.

$$
I G\left(f_{j}\right)=-\sum_{k=1}^{n} p\left(y_{k}\right) \log p\left(y_{k}\right)+\sum_{v l_{j} \in v_{j}} p\left(v a l_{j}\right) \times \sum_{k=1}^{n} p\left(y_{k} \mid v a l_{j}\right) \log p\left(y_{k} \mid v a l_{j}\right),
$$


where $n$ stands for the number of personalities; $v^{a l} l_{j}$ represents a certain value of feature $f_{j}$, while $V_{j}$ represents the value set of feature $f_{j}$; $\mathrm{p}\left(y_{k}\right)$ represents the probability that personality tag $y_{k}$ appears in dataset; $v a l_{j}$ represents the probability that feature $f_{j}=v a l_{j}$ appears in dataset; $\mathrm{p}\left(y_{k} \mid v a l_{j}\right)$ represents the probability that personality tag $y_{k}$ appears in dataset when feature $f_{j}$ equals to $v a l_{j}$. The distance between test user $t$ and user $i$ in training dataset is calculated with Eq. 6.

$$
d\left(x_{t}, x_{i}\right)=\sum_{j=1}^{r} \text { Weight }_{j} \times\left\|x_{t}^{j}-x_{i}^{j}\right\|,
$$

where $x_{t}^{j}$ and $x_{i}^{j}$ represent feature $f_{j}$ of test user $t$ and user $i$ in training dataset separately; Weight ${ }_{j}$ represents weight of feature $f_{j}$, while, as we employ entropy based weight method to compute distance, Weight $_{j}$ equals to $1 / r$ here; $\|\bullet\|$ denotes absolute value of returned real number. The calculation of distance is based on equivalent weight of features, which scales down less relevant features in the Euclidean axes. The algorithm is shown below.

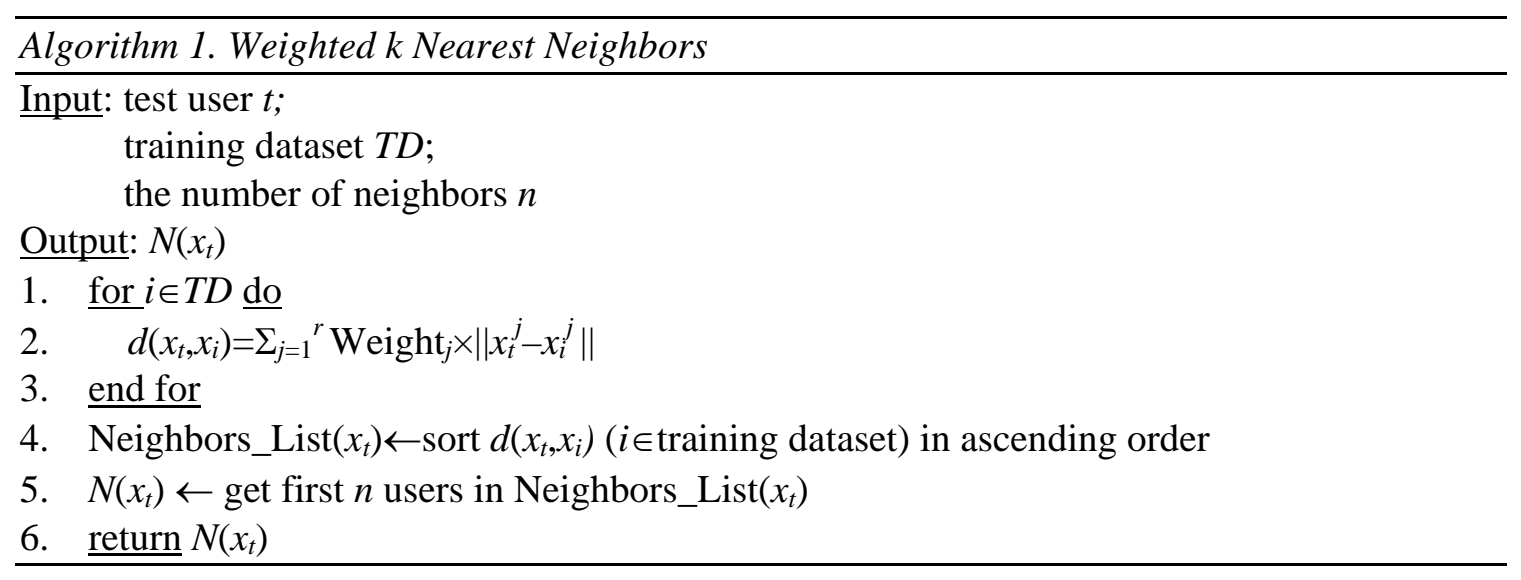

Predicting Users' Personality based on ML-kNN Model. The weighted ML-kNN model for predicting users' personalities, which is adapted from [10], is shown as follows.

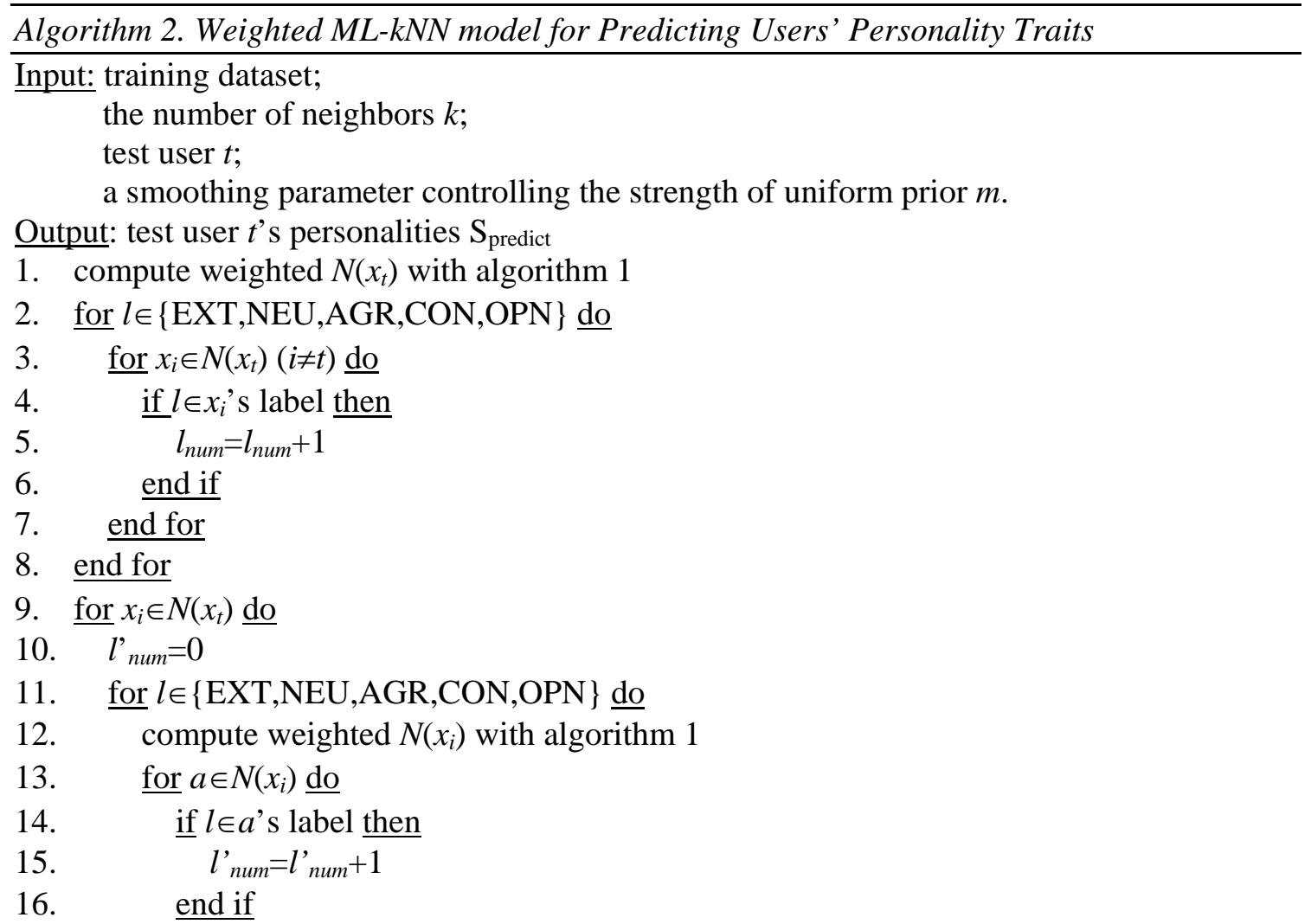


17. end for

18. if $l \in X_{i}$ 's label and $l_{\text {num }}{ }_{\text {num }}==l_{\text {num }}$ then

19. $\quad l_{\text {sum }}=l_{\text {sum }}+1$

20. else if $l \notin x_{i}$ 's label and $l_{\text {num }}==l_{\text {num }}$ then

21. $\quad \neg l_{\text {sum }}=\neg l_{\text {sum }}+1$

22. end if

23. end for

24. end for

25. $\overline{P\left(H_{1}{ }^{l}\right)}=\left(s+l_{\text {num }}\right) /(s \times 2+m) ; P\left(H_{0}{ }^{l}\right)=1-P\left(H_{1}{ }^{l}\right)$

26. $P\left(E_{j}^{l} \mid H_{1}^{l}\right)=\left(s+l_{\text {sum }}\right) /\left(s \times(k+1)+l_{\text {num }}\right)$

27. $P\left(E_{j}^{l} \mid H_{0}{ }^{l}\right)=\left(s+\neg l_{\text {sum }}\right) /\left(s \times(k+1)+m-l_{\text {num }}\right)$

28. $S_{\text {predict }} \leftarrow \arg \max _{b \in\{0,1\}} P\left(H_{b}{ }^{l}\right) P\left(E_{j}^{l} \mid H_{b}{ }^{l}\right)$

29. return $S_{\text {predict }}$

According to [10], the event that $t$ has personality $l$ is represented by $H_{1}{ }^{l}$, while the event that $t$ dosn't has personality $l$ is represented by $H_{0}{ }^{l} ; E_{j}{ }^{l}(j \in\{0,1, \ldots, k\})$ stands for the event that among the $k$ nearest neighbors of $t$, there are exactly $j$ instances which have personality $l$.

\section{Experiment and Evaluation}

Dataset. Personality traits are commonly expressed using five dimensions: EXT (extraversion), NEU(neuroticity), AGR(agreeableness), CON(conscientiousness), OPN(openness). Essays [11] is a large dataset of stream-of-consciousness texts (about 2400, one for each user), collected between 1997 and 2004 and labeled with personality classes. Texts have been produced by students who took the Big-5 test. The labels, that are self-assessments, are derived through z-scores computed by Mairesse et al [12] and converted from scores to nominal classes by us with median split. Since this corpus has been used by different scholars [12,13], it has been included in the shared task as a reference to previous works.

Weighted ML-kNN Classification. On the basis of information entropy theory, we calculate the unbalanced weights of features shown in Table 1.

Table 1. Weights of features

\begin{tabular}{|l|l|l|l|}
\hline \multicolumn{1}{|c|}{ Feature } & \multicolumn{1}{c|}{ Weight } & \multicolumn{1}{c|}{ Feature } & \multicolumn{1}{c|}{ Weight } \\
\hline CC & 0.027831087744011905 & VB & 0.029400552391457974 \\
\hline CD & 0.02672996424430056 & VBD & 0.025643899880597544 \\
\hline DT & 0.029758456002946024 & VBG & 0.02417241140121573 \\
\hline EX & 0.03017380368734125 & VBN & 0.023472627251283764 \\
\hline FW & 0.024390252225309453 & VBP & 0.03134910066504821 \\
\hline MD & 0.023628038225530442 & IN & 0.031015555198166418 \\
\hline NN & 0.03236128477834888 & J & 0.02544288143624376 \\
\hline NNS & 0.01920016075292616 & JJR & 0.021902027192898844 \\
\hline NNP & 0.028360053254371295 & JJS & 0.025614270721750324 \\
\hline NNPS & 0.025828148558535914 & WDT & 0.022229268510149624 \\
\hline PDT & 0.021052095077153526 & WP & 0.02377011942204686 \\
\hline POS & 0.020592460748347193 & WRB & 0.023793616698004654 \\
\hline PRP & 0.0312581791604354 & PERIOD & 0.03062577163640338 \\
\hline RB & 0.028407651862704004 & EXCLAMATION & 0.02354746317144375 \\
\hline RBR & 0.02128185054618292 & QUESTION & 0.02532700239203083 \\
\hline RBS & 0.031278905210584323 & OPSCORE & 0.04410245513329516 \\
\hline RP & 0.025666903200739062 & MISCORE & 0.04638371313119664 \\
\hline SYM & 0.02085859049540904 & AGSCORE & 0.032295211382231154 \\
\hline UH & 0.021254166609358104 & \multicolumn{2}{|l}{} \\
\hline
\end{tabular}


Then, we run the weighted ML-kNN algorithm to predict the personality traits of test users. Finally, we calculate the precision, recall and F-measure with $k$ ranging from 5 to 180. The F1-meature for different parameter $k$ is shown in Fig. 1.

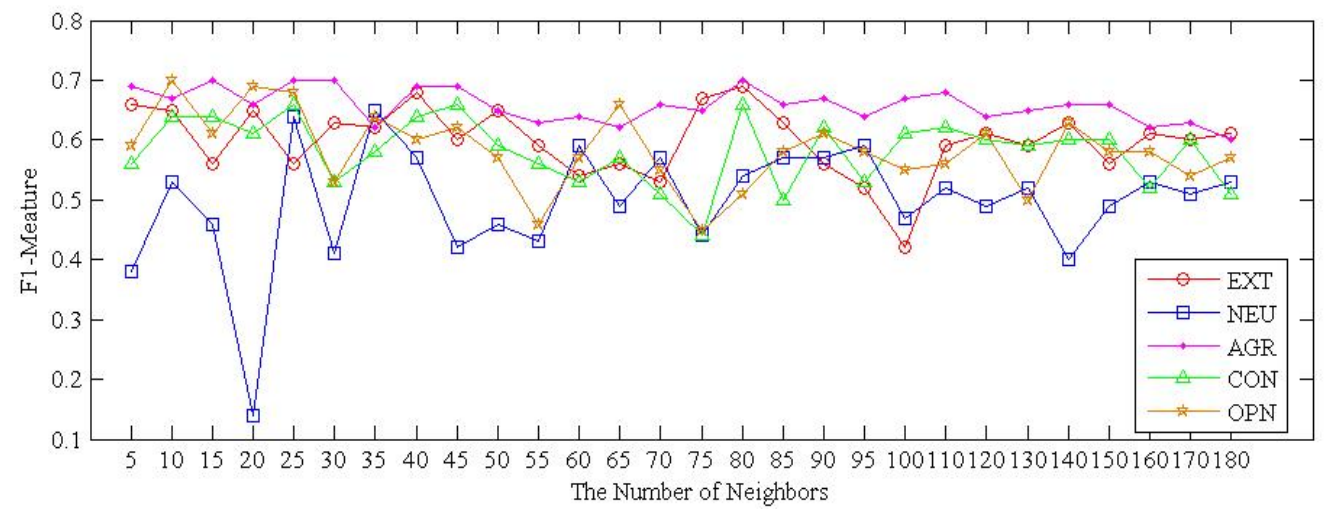

Figure 1. The F1-meature on the BIG-5 personality traits

The Workshop on Computational Personality Recognition (Shared Task) recommends participants to evaluate features and learning techniques, and compare the performances of their systems for personality recognition on a common benchmark. In this paper we compare our work with existing works on the standard Essays dataset in terms of average precision, recall and F1-measure. The result is shown in Table 2, from which we can observe that the recall of our method is significantly greater than existing methods, and as such, we get the best F1 measure.

Table 2. Comparison between our work and others' in average

\begin{tabular}{|c|c|c|c|c|}
\hline Related Works & Methods & Precision & Recall & F1-measure \\
\hline Our Work & Weighted ML-kNN & 0.55 & 0.89 & 0.65 \\
\hline$[4]$ & SVM & 0.55 & 0.55 & 0.55 \\
\hline$[5]$ & SVM,kNN,NB & 0.62 & 0.62 & 0.57 \\
\hline$[7]$ & SVM & NA & NA & 0.57 \\
\hline$[9]$ & NB & 0.53 & NA & NA \\
\hline
\end{tabular}

\section{Summary}

In this paper, we explored the feasibility of modeling user personality traits based on weighted ML-kNN. The reason behind our initiative in adopting ML-kNN, instead of classical kNN, is that there exist certain correlations among the Big-5 personality traits, and as such it is inappropriate to build 5 independent classifiers, one for each personality trait. We reported system performances in terms of precision, recall and F1-meature, and observed the expected positive effects.

Central to personality modeling are feature recognition and classification scheme. The features extracted and weighted in our method are based on information gain and information entropy, and the classifier is based on ML-kNN. It has been recognized that emotional features have great impact on user personality [7]. In this paper, we divide emotions into 3 categories: positive, neutral, and negative. We recognize that this partition is too coarse, and further research will be devoted to more fine-grained division of user emotions. Another ongoing work is to build ensemble of classifiers to improve precision. 


\section{References}

[1] D. Markovikj, S. Gievska, M. Kosinski D. Stillwell, Mining Facebook Data for Predictive Personality Modeling. Proc of Workshop on Computational Personality Recognition, AAAI Press, Melon Park, CA, 2013. http://clic.cimec.unitn.it/fabio/wcpr13/markovikj_wcpr13.pdf.

[2] F. Alam, Stepanov, A. Evgeny, G. Riccardi, Personality Traits Recognition on Social Network-Facebook, Proc of Workshop on Computational Personality Recognition, AAAI Press, Melon Park, CA, 2013, 6-9.

[3] J.M. McGloin, D.S. Kirk, An overview of social network analysis. Journal of Criminal Justice Education, 21(2), 2010, 169-181.

[4] B. Verhoeven, W. Daelemans, T.D. Smedt, Ensemble Methods for Personality Recognition, Proc of Workshop on Computational Personality Recognition, AAAI Press, Melon Park, CA, 2013, 35-38.

[5] G. Farnadi, S. Zoghbi, M.F. Moens, M.D. Cock, Recognising Personality Traits using Facebook Status Updates. Proc of Workshop on Computational Personality Recognition, AAAI Press, Melon Park, CA, 2013. http://clic.cimec.unitn.it/fabio/wcpr13/farnadi_wcpr13.pdf.

[6] T.M. Tomlinson, D. Hinote, D.B. Bracewell, Predicting Conscientiousness through Semantic Analysis of Facebook Posts. Proc of Workshop on Computational Personality Recognition, Press, Melon Park, CA, 2013, 31-34.

[7] S. Mohammad, S. Kiritchenko, Using Nuances of Emotion to Identify Personnality, Proc of Workshop on Computational Personality Recognition, AAAI Press, Melon Park, CA, 2013,27-30.

[8] D.S. Appling E.J. Briscoe, H.Hayes R.L. Mappus, Towards Automated Personality Identification using Speech Acts. Proc of Workshop on Computational Personality Recognition, AAAI Press, Melon Park, CA, 2013, 10-13.

[9] F. Iacobelli, A. Culotta, Too Neurotic, not too Friendly: Structured Personality Classification on Textual Data, Proc of Workshop on Computational Personality Recognition, AAAI Press, Melon Park, CA, 19-22.

[10] M.L. Zhang, Z.H. Zhou. ML-kNN: A lazy learning approach to multi-label learning, Pattern Recognition, 40(7), 2007, 2038-2048.

[11] J.W. Pennebaker, L.A. King, Linguistic styles: Language use as an individual difference, Journal of Personality and Social Psychology, 77(1999)1296-1312.

[12]F. Mairesse, M.A. Walker, M.R.Mehl, R.K.Moore, Using Linguistic Cues for the Automatic Recognition of Personality in Conversation and Text. Journal of Artificial intelligence Research, 30(2007)457-500.

[13] S. Argamon, S. Dhawle, M. Koppel, J.W. Pennebaker, Lexical Predictors of Personality Type. In: Proc. of Joint Annual Meeting of the Interface and the Classification Society of North America, St. Louis, MO, 2005, 1-16.

\section{Acknowledgment}

This work is supported by the National Natural Science Foundation of China under grant No.60973040; the Scientific and Technological Break-through Project of Jilin Province under grant No.20130206051GX; and the National Student Innovation Practice Program. 